THIS PAPER MUST BE CITED AS:

Proceedings of SPIE, Vol. 5840, p516-522 (2005)

\title{
LIGHT EMITTING DEVICES BASED ON NANOSTRUCTURED SEMICONDUCTORS
}

\author{
D. E. Gallardo, C. Bertoni and S. Dunn. \\ Nanotechnology Group, SIMS, Cranfield University, Beds, MK43 0AL. UK
}

\begin{abstract}
Light emitting devices based on high-efficiency photoluminescence (PL) fluorescent nanocrystals have been investigated in terms of the generation of light from the structure using a variety of deposition methods. An automated modified layer-by-layer ( $\mathrm{LbL}$ ) self-assembly technique has been employed to produce multilayers of thiol-capped red fluorescing CdTe nanocrystals. Indium-tin-oxide (ITO) and aluminium electrodes were used as the electrodes. Morphological characterization was carried out through Schottky field effect (SFEG) SEM and atomic force microscopy (AFM). The structures built presented clear red electroluminescence (EL) to the naked eye. Turn on voltages were found to be in the range of 3-6 volts while the onset current was in the order of tens of microamperes. The role of structure homogeneity, the presence of pinholes and lifetime extension were features addressed during this investigation. Samples with a lifetime of continuous operation in air longer than 60 minutes and highly stable EL spectra were achieved; EL was visible to the unaided eye, although the brightness was still below the commercial standards and has not yet been qualified.
\end{abstract}

\section{INTRODUCTION}

\section{Quantum size effects in semiconductors}

When solids are reduced in size to the nanometric scale, the Coulomb interaction felt by the electrons is notably affected. As a consequence, the energy bands rearrange into new configurations, leading to new material properties. These are known as quantum size effects (QSE) [1].

The most relevant QSE on nanoscaled semiconductors for light emission applications is a size-dependant band-gap widening. The enlargement of the prohibited band shifts the associated emission wavelength towards more energetic parts of the electromagnetic spectrum. As a result, semiconductors whose bulk emission wavelength was in the infrared are open to visible radiation when in the nanoscale. A well documented example is CdTe, whose bulk band-gap is 
$1.56 \mathrm{eV}$ and emits in the infrared; for nanometric size particles, emissions in the whole visible spectrum have been reported [2-6].

Another important QSE is the splitting of the lower exciton state for electrons and holes into different levels of different spin configuration with an energy difference in the order of a few $\mathrm{meV}[2,7]$. Thermal activation at room temperature is then enough to fill these states with carriers. As a consequence, the radiative recombination is no longer restricted by the spin statistics that set a theoretical limit of $25 \%$ efficiency. Therefore the radiative efficiency in such devices can be much higher than $25 \%$ with reports of $80 \%$ common place in the literature.

\section{Nanostructured semiconductor light emitting devices (NSLED)}

NSLEDs have been thoroughly investigated in the last decade. High PL efficiency have been reported for nanocrystals of several types of semiconductors $[4,8]$. This high PL efficiency encouraged researchers to try to electrically stimulate light emission with a high EL yield. A large range of semiconductors have been employed in NSLEDs: CdTe, CdSe, $\mathrm{CdS}, \mathrm{ZnS}$ are amoung the most common although others have been used. Different nanocrystals deposition techniques have been employed (spin-coating, layer-by-layer, dip-coating...) along with different suspension solvents to generate the active layer. The active layer has to be homogeneous and free from defects if the device is to have a good overall efficiency.

A typical device structure is a sandwich of a single or multilayer of nanocrystals between the chosen electrodes. ITO has been traditionally used as transparent anode, due to its high work function that improves hole injection. Low work function metals like $\mathrm{Al}, \mathrm{Ca}$ and $\mathrm{Mg}$ have been used as the cathode. These increase the electron injection into the active layer. [9] However, so far low EL efficiencies have been obtained [5,10], another problem is that the device lifetime remains an issue $[2,11,12]$. The presence of pinholes is also a common problem in the manufacturing of these structures, leading to abnormally high current density levels that destroy the device or reduce their lifetime even further [13].

The use of an organic hole transport layer has been an extended practice for many groups. The role of this layer is to improve hole injection into the nanocrystals. Conducting polymers like PAni, PPV, PEDOT:PSS and others present a highest occupied molecular level (HOMO) that is energetically below the conduction level of ITO, providing a better matching with the semiconductor valence band $[4,5,10,11]$. However, a second but no less important role is the prevention of pinholes. Conductive polymers are normally deposited through spin-coating from organic solvent solutions, yielding well homogeneous layers with a thickness as high as $200-300 \mathrm{~nm}$. As a payback, the limitations of organic compounds are eventually added to the structure. Combinations of semiconductor nanoparticles and electroluminescent polymers have also been extensively studied as a way to stimulate either polymer or nanoparticle emission [6,12,14]. Although improved PL and/or EL have been found in some cases, new problems emerged associated to the interaction between both species. Additionally, electroluminescent polymers have frequently a high oxidation susceptibility, adding difficulty to the manufacture process and the operation of the devices. Finally, the introduction of organic materials within the structure posses some risks concerning thermal stability for the final device.

In this article, the results of the investigation of NSLEDs based on red CdTe nanocrystals is presented. No organic materials have been employed with an active part in light emission. The different problems presented so far by these devices have been directly addressed.

\section{EXPERIMENTAL}

Samples consisted in a stacked structure in which the active layer was sandwiched between the electrodes. The substrates were bits of $26 \mathrm{~mm} \times 18 \mathrm{~mm}$ cut from an ITO-coated glass sheet as supplied from Merk. The ITO on these cut pieces were covered with a steel mask and a cellulose laquer (from RS) was sprayed on the top. After the removal of the mask, laquer strips of either $2 \mathrm{~mm}$ or $4 \mathrm{~mm}$ width protected the underlying ITO. The substrates were then etched using hydrochloric acid $60 \%$ Vol. in water at $50^{\circ} \mathrm{C}$ for 5 minutes to remove the unprotected ITO, leaving only the ITO strips on the substrates. A thorough ultrasonic cleaning with isopropanol and acetone, and a final baking at $140^{\circ} \mathrm{C}$ for 12 hours 
completed the substrate preparation. High PL efficiency thiol-capped CdTe nanocrystals were deposited onto the substrates from a colloidal water solution. The size of the nanocrystals was around $3 \mathrm{~nm}$. For this step a modified a layer-by-layer (LbL) $[15,16]$ technique was used. The samples were submerged in a commercial water solution of $20 \%$ poly-(diallyldimethylammonium) (PDDA) diluted to $0.5 \%$. Then, the samples were rinsed for 5 min in distilled water and immersed in the CdTe solution for $10 \mathrm{~min}$. This was followed by another $5 \mathrm{~min}$ rinsing [17]. The process was repeated until the required number of layers was deposited. This procedure was done either by hand or using a robotized arm. For the robotized procedure, a slow withdrawing of the samples from the solutions was programmed for an efficient solvent removal. Aluminium electrodes were deposited through a steel shadow mask using a thermal evaporator from Edwards. The electrodes were $4 \mathrm{~mm}$ width, forming active areas of either $8 \mathrm{~mm}^{2}$ or $16 \mathrm{~mm}^{2}$ where they crossed with the underlying ITO strips.

A Digital instruments DI3000 AFM system operating in tapping mode was used for surface characterization. Ultra high precision SEM micrographs of device cross sections were taken using a field emission gun (SFEG) XL30 Philips SEM. Thickness and roughness measurements were assisted by a Veeco Dektak profilometer. .Absorbance measurements were carried out with a Lambda 7 UV-Vis spectrometer from Perkin-Elmer. PL spectra were obtained with a Fluorolog 3 spectrofluorimeter with a $450 \mathrm{~W}$ xenon lamp, with $2 \mathrm{~nm}$ slits for excitation and detection, and an excitation light of $450 \mathrm{~nm}$. EL spectra were taken with the same equipment, blocking the light source and using a 10nm detector slit. PL inspection was carried out under a 356nm UV light.

\section{RESULTS AND DISCUSSION}

Absorbance measurements were carried out on CdTe colloidal solutions diluted in water, as a preliminary stage. The resulting data is presented in figure 1. Taking into account that CdTe is a direct band-gap semiconductor, the energy band-gap can be calculated through the proportionality [18]:

$$
(A \cdot E)^{2} \propto\left(E-E_{G}\right)
$$

where $\mathrm{A}$ is the measured absorption, $\mathrm{E}$ is the excitation energy and $\mathrm{EG}$ is the bang-gap value. The calculated value for

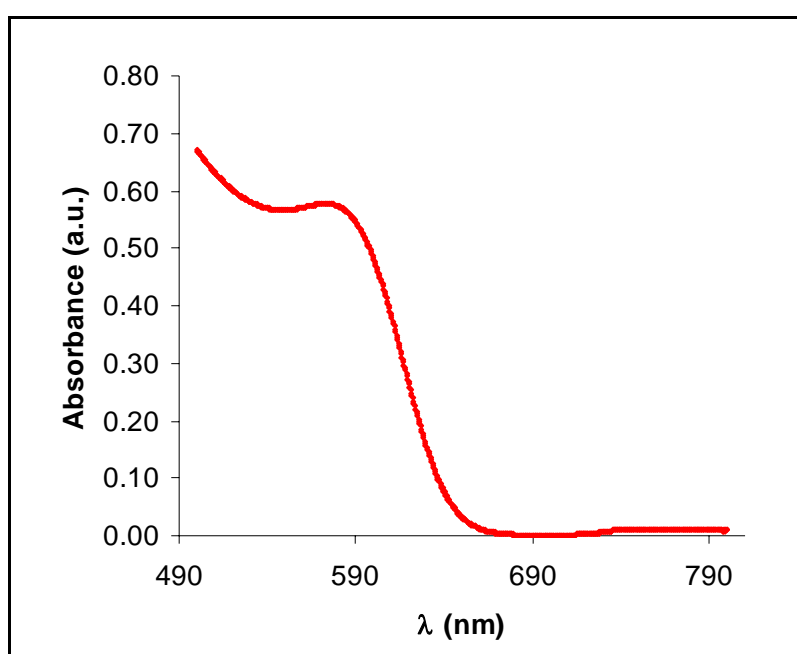

Fig.1. Absorption spectrum of CdTe nanocrystals in this last parameter was $\mathrm{E}_{\mathrm{G}}=2.0 \mathrm{eV}$. According to refs. $[19,20]$, it would correspond to a particle size around 2.5 $3.5 \mathrm{~nm}$, matching the data from the nanocrystals manufacturing.

In the first stage of research, devices of 1 to 10 layers were prepared using a manual LbL procedure following ref. [2]. The use of organic poly-electrolytes does not pose a threat on the device performance, because these polymers are used as a gluing passive element, taking no part in light emission.

Cross sections and surface analyses were carried out on these samples using SFEG and AFM microscopes. The surface images revealed a very porous structure (fig.2), with the nanoparticles arranged in clusters of $15-20 \mathrm{~nm}$. With a nanoparticle size in solution in the range of $2 \mathrm{~nm}$ to $5 \mathrm{~nm}$, it was concluded that the nanoparticles were agglomerating during the deposition stage. Cross section
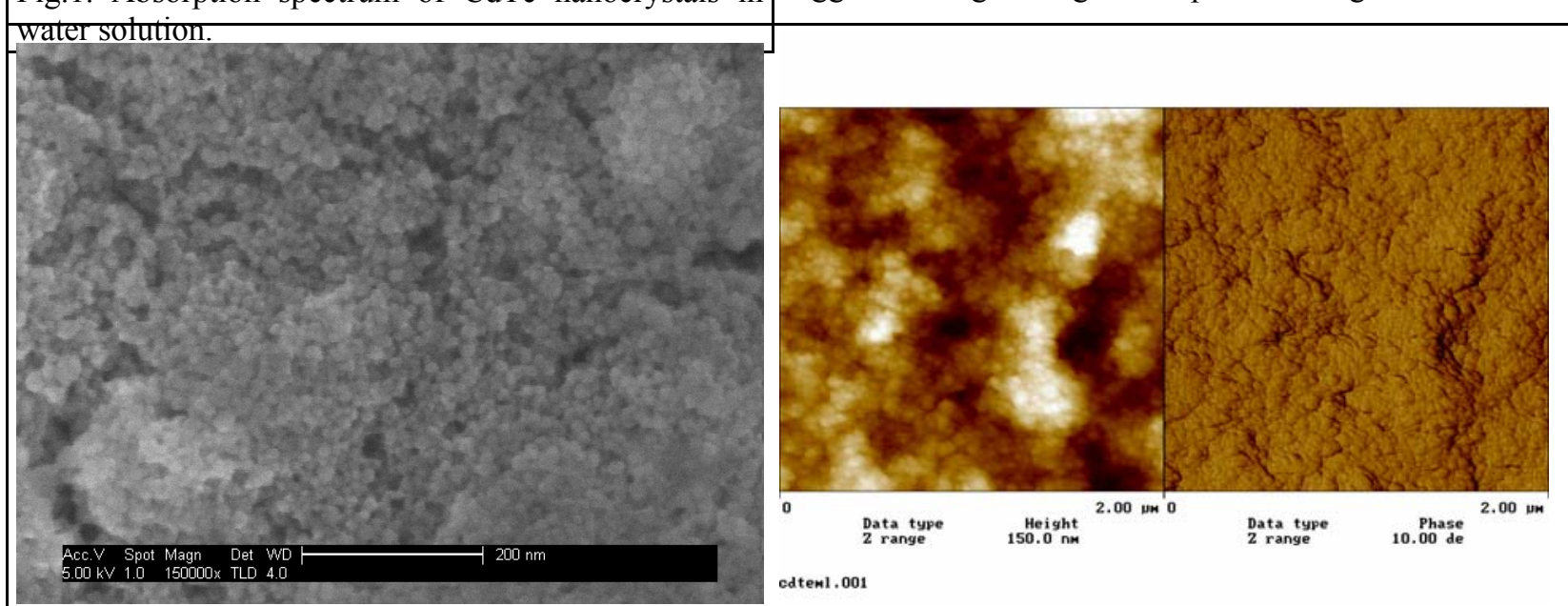

Fig.2. SFEG and AFM images of the multilayer surface. The high porosity of the multilayer favours the development of pinholes. 
images showed a very rough surface (fig.3), presumably due to the porosity of the CdTe multilayers. The thicknesses of a monolayers were around $30 \mathrm{~nm}$. Profilometer scans supported those data, summarized in table 1.

\begin{tabular}{|c|c|c|c|}
\hline \multicolumn{4}{|c|}{ Table 1. LbL methods comparison } \\
\hline Method & Roughness & Thickness & Thickness (per monolayer) \\
\hline Manual (10 layers) & $100 \mathrm{~nm}$ & $300 \mathrm{~nm}$ & $30 \mathrm{~nm}$ \\
\hline Automated (50 layers) & Below resolution & $150 \mathrm{~nm}$ & $3 \mathrm{~nm}$ \\
\hline
\end{tabular}

$\mathrm{I}-\mathrm{V}$ measurements revealed currents densities of the order of $100 \mathrm{~mA} / \mathrm{cm}^{2}$ in most of the samples even at low voltages $(1.0-2.0 \mathrm{~V})$, until electric failure which was signalled by current spikes and the device sparking. Prompt PL loss was revealed under UV light, due to the developing of heat within the structure. Little success was attained in EL, where emission faded out rapidly ( 5 minutes maximum) without allowing for EL spectrometry. The light, though faint, was visible for the naked eye and clearly red. These results indicated that the development of pinholes during the top electrode deposition induced high short-circuiting currents that eventually destroyed the samples and prevented light emission. The abundance of pinholes was related to the structural porosity observed under the microscopes. The fast lost of EL was related to the introduction of oxygen within the structure. The LbL takes place in air, due to the high stability of the nanoparticles. Ulterior long vacuum exposure helps to remove the air and moisture that could remain in the sample. But porosity makes this process more difficult and, as a result, the lifetime of the device is considerably

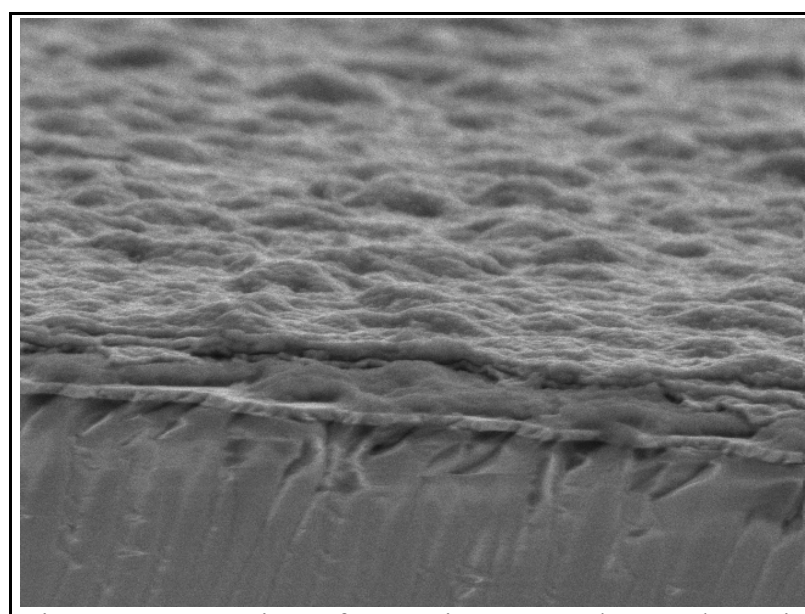

Fig.3. Cross section of an active area. The top layer is aluminium, followed by a thick area, the CdTe multilayers. The ITO is distinguishable as a thin layer right over the glass.

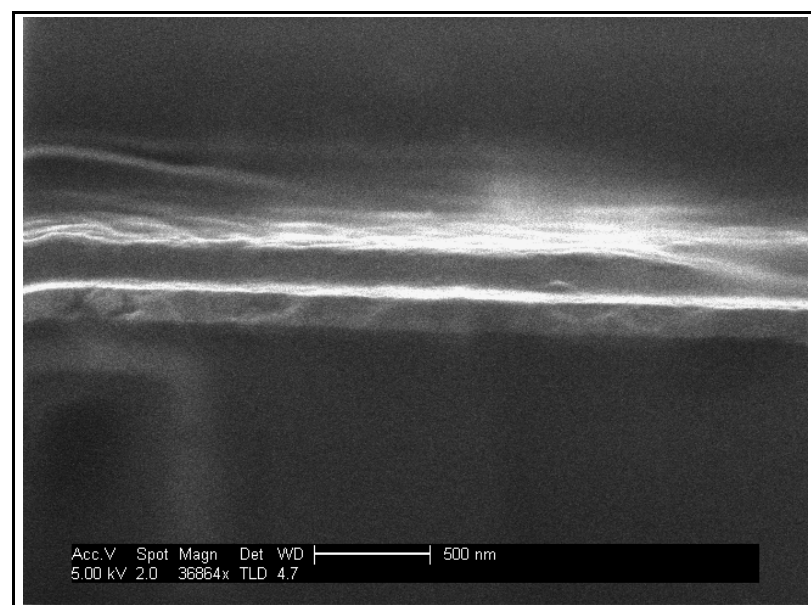

Fig.4. Cross section of a stack of 50 CdTe nanoparticle layers deposited by a robotic arm. shortened. To overcome the pinholes issue without using additional components in the device, the LbL stage demanded a significant improvement.

Poly-ethylenimine (PEI) was used in the LbL process as the first polyelectrolyte, favouring the adhesion to the substrate surface, while PDDA is used for subsequent layers. It has been reported [17] that PEI molecular chains are keen to form filaments that hang out off the surface; these filaments can surround a number of particles forming a protruding agglomerate that favours further growth. On the other hand, PDDA tends to form more homogeneous layers and no filament formation have been reported. Thus, PEI was excluded from subsequent the LbL process. Samples were prepared with equal numbers of layers, with and without PEI as a first polyelectrolyte, to test the adhesion of CdTe by absorbance measurements. The results of comparative absorbance indicated that the suppression of PEI did not affect the amount of CdTe attached to the substrates. Additionally, a manual LbL

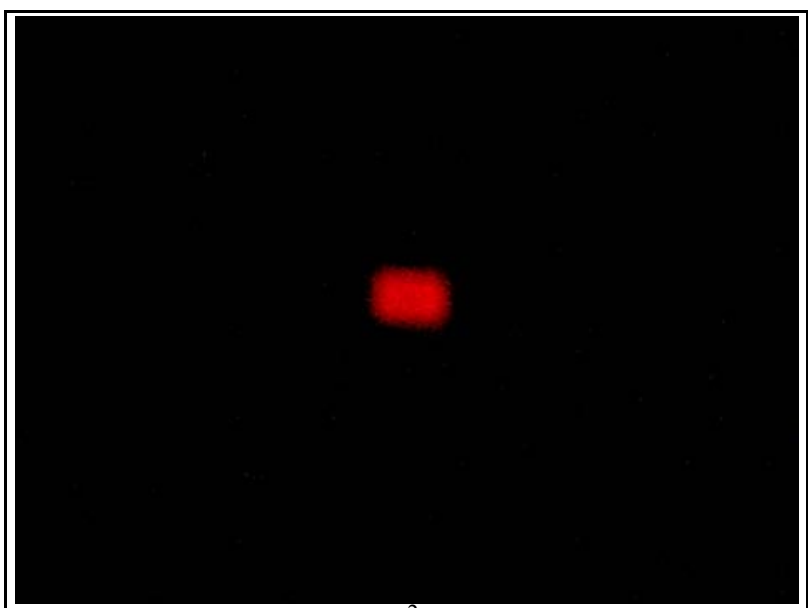

Fig.5. An $8 \mathrm{~mm}^{2}$ emitting area. 
process leads to very inhomogeneous deposition, with an added risk of scratching of the samples. Moreover, the process is long and time consuming. A robotic arm was programmed to carry out the LbL process. Figure 4 shows a cross section of a $50 \times$ CdTe multilayer produced by the robot, with an overall thickness of $150 \mathrm{~nm}$. The $150 \mathrm{~nm}$ thick ITO layer, observable below the CdTe, gives an idea of the thickness of the active layers, that results in a value around $3 \mathrm{~nm}$ per layer. This number matched the previous data about the size of the nanoparticles. Also, it indicated that the different CdTe layers were actually monolayers of nanoparticles, rather than a porous stack of nanoparticles aggregates. A dramatic improvement in roughness at long scales can be also appreciated. Data is summarized in table 1, comparing manual and automatic depositions.

Samples with 10 layers of CdTe produced through the automated method were covered with aluminium electrodes. An Al minimum thickness of 100nm was always employed to prevent oxidation. The samples showed resistivities over $1 \mathrm{~K} \Omega$ in every area, constituting a first confirmation of the efficient removal of pinholes. It was possible to obtain EL visible to the eye from several areas (see fig.5), with a turn on voltage around $3 \mathrm{~V}$ and current densities between 375 $700 \mathrm{~mA} / \mathrm{cm}^{2}$. However, the EL was always lost after 5-10 minutes of operation.

Additionally, a significant loss of PL was detected after Al deposition on many areas under UV-lamp inspection. This PL loss was later followed by a total absence of EL. A problem due to thermal effects during Al deposition was judged to be a possible reason for the loss of PL. The heat radiated by the filaments during the evaporation could be heating the samples to the point of chemical degradation of the CdTe nanoparticles. In consequence, the distance between the samples to be coated and the filaments within the chamber was doubled to around $30 \mathrm{~cm}$. Newly produced samples showed an improved PL under UV-light, supporting the hypothesis of PL loss by heating. Typical normalized EL and PL spectra are showed in figure 6. As can be seen, there was a steady growth of EL intensity with voltage, until a maximum was achieved (6V in figure 6), marking an optimal voltage. If the voltage was increased further, little or none

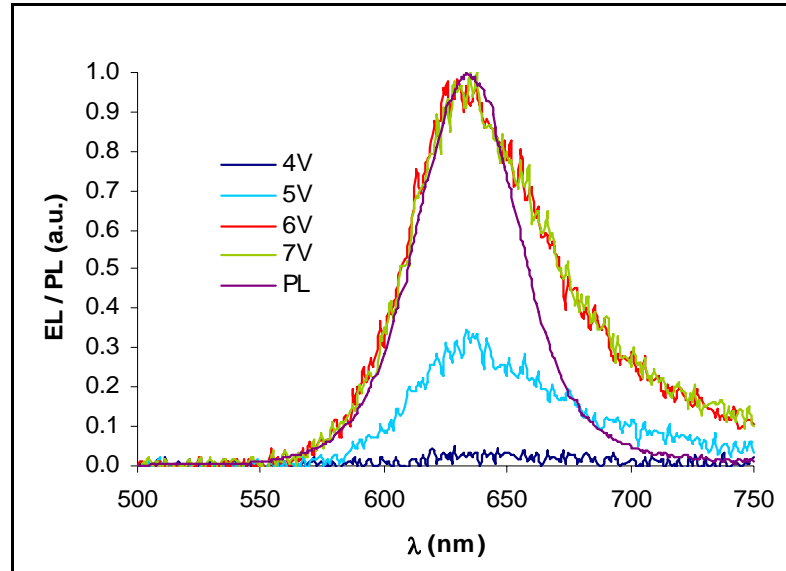

Fig.6. EL behaviour for a range of voltages. Optimal voltage is $6 \mathrm{~V}$ with a maximum EL. The PL is also shown for comparison.

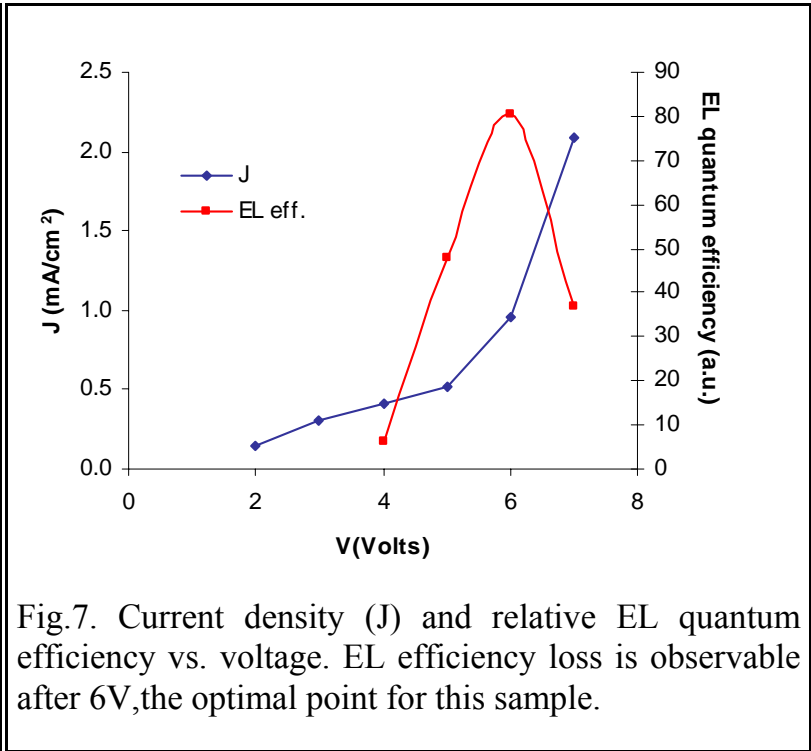

higher EL intensity was obtained (7V in figure. 6). This fact is clearly shown in figure 7, where the relative EL quantum efficiency has been estimated dividing the integrated EL intensity by the corresponding current density [6,21]. If this overloading voltage, higher than the optimal value, was kept a few tens of seconds, the samples rapidly lost their EL while a sudden increase of several orders in current could be detected.

The reason for this behaviour still needs further investigation. However, the hypothesis of electronic avalanche seems plausible. Due to the thinness of the active layers, the electric fields into these samples can reach values of around $10^{6} \mathrm{~V} / \mathrm{cm}$. At these high fields the electrons could have enough energy to free additional electrons by collision (scattering). The new freed electrons can free additional electrons in an avalanche effect. EL was recoverable if the overload time lasted only a few seconds, supporting the avalanche hypothesis. Longer periods under current overload irreversibly destroyed the emitting areas, probably by local heating. The avalanche hypothesis for EL loss at voltages higher than the optimal point can be investigated by varying the number of layers within the structure. The electron 
avalanche is normally triggered when the electric field reaches a critical value, characteristic of each semiconductor (e.g. Si with $2 \times 10^{5} \mathrm{~V} / \mathrm{cm}$ ). If the rupture point were detected at the same field for different sample thicknesses, it would be a definitive support for this hypothesis.

Another finding was a dramatically extended lifetime in air for these new samples. An optimal value of voltage was applied to an active area and kept constant for an hour, while taking EL spectra every 5 minutes. These analyses were carried out on several areas of different samples, under typical laboratory temperatures and atmosphere, with results similar to the one shown in fig 8. An initial fluctuation of the EL intensity was observable during the first 10-15 minutes, after which the EL relatively stabilized (fig 9). The reasons for the extended lifetime are thought to be entirely related with the modified processing of the samples. As has been discussed previously, the lower porosity of the nanocrystals layers reduced the density of pinholes. This reduction could push local fields below the avalanche level. Another factor seemed to be related with the new conditions for the Al deposition. The reduced thermal damage over the nanocrystals could have helped to preserve their organic coating, extending their lifetime. Additionally, the thickness of the $\mathrm{Al}$ electrodes, over $150 \mathrm{~nm}$, is probably preventing any oxidation of the nanoparticles during operation in air.

Although standard irradiance measurements have not yet been performed, it was clear by eye inspection that the brightness of these samples was still low in comparison with other light emission mechanisms. Despite very high EL quantum efficiencies are theoretically possible with these devices, low brightness remains a problem for reasons still unknown. Other kind of analyses are open for research now that the operational lifetime in air has been successfully extended.
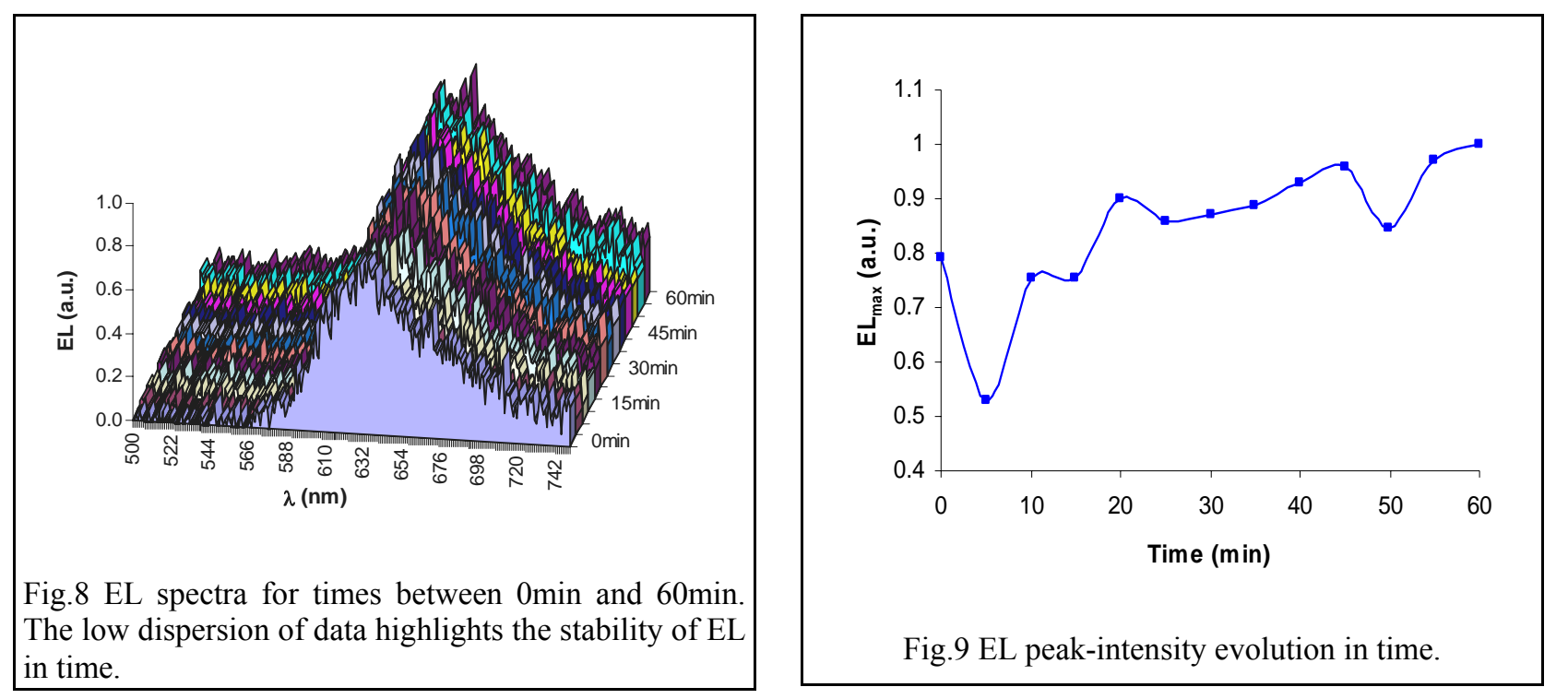

\section{CONCLUSSIONS}

NSLEDs production processes have been optimized in different ways for improved manufacturing and electrical performances. High homogeneity and low roughness have been achieved with automated and controlled deposition of semiconductor nanoparticle layers, leading to improved electrical characteristics. All the deposition process is carried out in normal laboratory conditions, eliminating complicated and expensive controlled environment requirements. Additionally, the production yield (the proportion of valid samples within a batch) has grown over the $90 \%$. Continuous stable operation in air have been demonstrated through the constancy of electrical and luminescence variables. As a result of the in-air extended lifetime operation, further analysis are now possible to address remaining issues that before were impossible to be carried out because of the fast degradation of the samples. The brightness of the samples, as an example, needs to be improved further for commercial applications. 


\section{ACKNOWLEDGEMENTS}

We would like to thank Dr N.P. Gaponik, from the Institute of Physical Chemistry of the University of Hamburg, for the invaluable help provided.

\section{REFERENCES}

1. Weller, H. (1993), Adv. Mat., Vol. 5, No. 2, pp. 88-95.

2. Gao, M., Lesser, C., Kirstein, S., Mohwald, H., Rogach, A.L. and Weller, H. (2000), J. Appl. Phys., Vol. 87(5), pp. 2297-2302.

3. Gaponik, N.P., Talapin, D.V., Rogach, A.L., Hoppe, K., Shevchenko, E.V., Kornowski, A., Eychmüller, A. and Weller, H. (2002), J. Phys. Chem. B, Vol. 106, pp. 7177-7185.

4. Talapin, D.V., Poznyak, S.K., Gaponik, N.P., Rogach, A.L. and Eychmuller, A. (2002), Physica E, Vol. 14, pp. 237-241.

5. Mattoussi, H., Radzilowski, L.H., Dabbousi, B.O., Thomas, E.L., Bawendi, M.G. and Rubner, M.F. (1998), J. Appl. Phys., Vol. 83(12), pp. 7965-7974.

6. Gaponik, N.P., Talapin, D.V. and Rogach, A.L. (1999), Phys. Chem. Chem. Phys., Vol. 1, pp. 1787-1789.

7. Efros, Al.L. and Rosen, M. (1998), Phys. Rev. B, Vol. 58, No. 11, pp. 7120-7135.

8. Gaponenko, S. V. Optical properties of semiconductor nanocrystals. Cambridge, UK: Cambridge University Press; 98.

9. Ginger, D.S. and Greenham, N.C. (2000), Journal of Applied Physics, Vol. 87(3), pp. 1361-1368.

10. Colvin, V.L., Schlamp, M.C. and Alivisatos, A.P. (1994), Letters to Nature, Vol. 370, pp. 354.

11. Chen, W., Grouquist, D. and Roark, J. (2002), J. Nanosci. Nanotech., Vol. 2, No. 1, pp. 47-53.

12. Gao, M., Richter, B., Kirstein, S. and Mohwald, H. (1998), J. Phys. Chem., Vol. 102, pp. 4096-4103.

13. Takamoto, D.Y., Aydil, E., Zasadzinski, J.A., Ivanova, A.T., Schwartz, D.K., Yang, T. and Cremer, P.S. (2001), Science, Vol. 293, pp. 1292-1295.

14. Dabbousi, B.O., Bawendi, M.G., Onitsuka, O. and Rubner, M.F. (1995), Appl. Phys. Lett., Vol. 66(11), pp. 13161318.

15. Decher, G. (1997), Science, Vol. 277, pp. 1232-1237.

16. Schmitt, J., Decher, G., Dressick, W.J., Brandow, S.L., Geer, R.E., Shashidhar, R. and Calvert, J.M. (1997), Adv. Mater., Vol. 9 (1), pp. 61-65.

17. Tang, Z., Wang, Y. and Kotov, N.A. (2002), Langmuir, Vol. 18, pp. 7035-7040.

18. Singh, J. Optoelectronics. An introduction to materials and devices. USA: McGraw-Hill; 96.

19. Vij, D. R., Vij, D.R. Handbook of electroluminescence materials. London, UK: IOP Publishing Ltd; 2004.

20. Tang, Z., Kotov, N.A. and Giersig, M. (2002), Science, Vol. 297, pp. 237-240.

21. Gaponik, N.P., Talapin, D.V., Rogach, A.L. and Eychmuller, A. (2000), J. Mate. Chem., Vol. 10, pp. $2163-2166$. 